\title{
Tetrahydrocurcumin Ameliorates Kidney Injury and High Systolic Blood Pressure in High-Fat Diet-Induced Type 2 Diabetic Mice
}

\author{
Weerapon Sangartit ${ }^{1,2,3}$, Kyung Bong Ha ${ }^{1, *}$, Eun Soo Lee ${ }^{1,4}$, Hong Min Kim5 Upa Kukongviriyapan ${ }^{2,3}$, \\ Eun Young Lee ${ }^{6}$, Choon Hee Chung ${ }^{1,4}$
}

${ }^{1}$ Department of Internal Medicine, Yonsei University Wonju College of Medicine, Wonju, Korea; ${ }^{2}$ Department of Physiology, Faculty of Medicine, Khon Kaen University; ${ }^{3}$ Cardiovascular Research Group, Khon Kaen University, Khon Kaen, Thailand; ${ }^{4}$ Institution of Genetic Cohort, Yonsei University Wonju College of Medicine, Wonju; ${ }^{5}$ Astrogen Inc., Daegu; ${ }^{6}$ Department of Internal Medicine and Institute of Tissue Regeneration, BK21 FOUR Project, Soonchunhyang University College of Medicine, Cheonan, Korea

Background: Activation of the intrarenal renin-angiotensin system (RAS) is implicated in the pathogenesis of kidney injury and hypertension. We aimed to investigate the protective effect of tetrahydrocurcumin (THU) on intrarenal RAS expression, kidney injury, and systolic blood pressure (SBP) in high-fat diet (HFD)-induced type 2 diabetic mice.

Methods: Eight-week-old male mice were fed a regular diet (RD) or HFD for 12 weeks, and THU (50 or $100 \mathrm{mg} / \mathrm{kg} /$ day) was intragastrically administered with HFD. Physiological and metabolic changes were monitored and the expression of RAS components and markers of kidney injury were assessed.

Results: HFD-fed mice exhibited hyperglycemia, insulin resistance, and dyslipidemia compared to those in the RD group $(P<0.05)$. Kidney injury in these mice was indicated by an increase in the ratio of albumin to creatinine, glomerular hypertrophy, and the effacement of podocyte foot processes. Expression of intrarenal angiotensin-converting enzyme, angiotensin II type I receptor, nicotinamide adenine dinucleotide phosphate (NADPH) oxidase-4, and monocyte chemoattractant protein-1 was also markedly increased in HFD-fed mice. HFD-fed mice exhibited elevated SBP that was accompanied by an increase in the wall thickness and vascular cross-sectional area $(P<0.05), 12$ weeks post-HFD consumption. Treatment with THU $(100 \mathrm{mg} / \mathrm{kg} /$ day $)$ suppressed intrarenal RAS activation, improved insulin sensitivity, and reduced SBP, thus, attenuating kidney injury in these mice.

Conclusion: THU alleviated kidney injury in mice with HFD-induced type 2 diabetes, possibly by blunting the activation of the intrarenal RAS/nicotinamide adenine dinucleotide phosphate oxidase IV (NOX4)/monocyte chemoattractant protein 1 (MCP-1) axis and by lowering the high SBP.

Keywords: Diabetic nephropathies; Renin-angiotensin system; Hypertension; Tetrahydrocurcumin; Curcumin

Received: 8 February 2021, Revised: 21 May 2021, Accepted: 25 May 2021

Corresponding author: Choon Hee Chung

Department of Internal Medicine, Yonsei University Wonju College of Medicine, 20 Ilsan-ro, Wonju 26426, Korea

Tel: +82-33-741-0506, Fax: +82-33-731-5884, E-mail: cchung@yonsei.ac.kr

\section{Copyright $(92021$ Korean Endocrine Society}

This is an Open Access article distributed under the terms of the Creative Commons Attribution Non-Commercial License (https://creativecommons.org/ licenses/by-nc/4.0/) which permits unrestricted non-commercial use, distribution, and reproduction in any medium, provided the original work is properly cited.

*These authors contributed equally to this work. 


\section{INTRODUCTION}

Hypertension is a risk factor for cardiovascular disease, and accelerates the progression of diabetic nephropathy (DN) [1]. The correlation between obesity and hypertension has been well established. Obesity activates the sympathetic nervous system and increases the activity of the renin-angiotensin system (RAS), leading to hypertension [2-4]. In addition, excessive fat accumulation induces hypercholesterolemia, which can damage the kidneys and promote atherosclerosis [5]. Abnormal lipid accumulation in branching vessels thickens the vascular walls and narrows the lumen, leading to increased peripheral resistance [6,7]. Moreover, obesity contributes to increased renal sodium reabsorption due to fat accumulation in the kidneys. Despite increased tubular reabsorption, renal vasodilation and glomerular hyperfiltration are increased to maintain sodium balance [8-10]. In $\mathrm{DN}$, glomerular hyperfiltration is the primary cause of glomerular damage, resulting in albuminuria [11]. Moreover, activation of the intrarenal RAS, increased sympathetic activity, oxidative stress, and increased activity of renal nicotinamide adenine dinucleotide phosphate oxidase IV (NOX4) is considered to be an underlying mechanism of DN [12]. Therefore, preventing hypertension and obesity is one way to prevent DN [13]. A previous study reported improved kidney function in non-diabetic patients with chronic kidney disease (CKD) after treatment with antihypertensive drugs [14]. Interestingly, albuminuria and blood pressure levels in type 2 diabetic patients reduced after 12 weeks of treatment with angiotensin-converting enzyme (ACE) inhibitor $[15,16]$. These results suggest that lowering blood pressure and RAS activity is a therapeutic strategy for preventing renal failure. Moreover, inhibition of oxidative stress has been shown to attenuate the symptoms of $\mathrm{DN}$, including albuminuria and renal sclerosis, in an experimental diabetic animal model $[17,18]$. Recently, in patients with CKD, new treatments using combinations of various drugs, such as antihypertensives, RAS inhibitors, and angiotensin II type I receptor blockers, have been investigated [19]. Tetrahydrocurcumin (THU), a major metabolite of curcumin (CUR), exhibits several pharmacological activities, including antihypertensive, antitumor, and antidiabetic properties by reducing lipid peroxide levels, fasting blood glucose levels, and hypertension [20-22]. In an experiment comparing the activities of CUR and THU, it was confirmed that the expression levels of cyclooxygenase 2 and nuclear factor- $\kappa \mathrm{B}$ were reduced more effectively by THU treatment than by treatment with CUR [23]. To date, the underlying mechanisms of THU in alleviating kidney injury have not been investigated in an obese or a diabetic animal model. Therefore, the aim of this study was to investigate the protective effect of THU on kidney injury and high systolic blood pressure (SBP) in high-fat diet (HFD)-induced type 2 diabetic mice. An animal model of type 2 diabetes with albuminuria and DN was induced by feeding HFD for 12 weeks [24], and was selected to investigate the protective effect of THU. Here, we hypothesized that THU might have a beneficial effect on kidney injury by lowering high blood glucose levels and preventing an increase in blood pressure.

\section{METHODS}

\section{Animal study}

Eight-week-old male C57BL/6J mice, weighing 20 to $25 \mathrm{~g}$, were purchased from Daehan Bio Link Co. (Eumseong, Korea). Mice were acclimatized for 7 days under constant room temperature $\left(22^{\circ} \mathrm{C} \pm 2{ }^{\circ} \mathrm{C}\right), 60 \% \pm 5 \%$ humidity, and a 12-hour light/ dark cycle. The mice had free access to water and were fed a regular diet $(\mathrm{RD})$. Thereafter, the mice were randomly divided into four groups ( $n=10$ in each group): RD with vehicle (RD+Veh), HFD with vehicle (HFD+Veh; HFD defined as diet providing $60 \%$ of its calories from fat; Research Diets Inc., New Brunswick, NJ, USA), HFD with THU 50 mg/kg/day (HFD+ THU50), and HFD with THU 100 mg/kg/day (HFD+THU100).

THU was intragastrically administered to the HFD-fed mice. Food intake and body weight of each mouse were measured weekly for the duration of the experiment. After 12 weeks, the serum and kidney tissues were collected and stored at $-80^{\circ} \mathrm{C}$ until further processing. All experiments were approved by the Institutional Animal Care and Use Committee of Yonsei University Wonju College of Medicine (YWC-170802-2).

\section{Metabolic parameter measurements}

At the end of the experiment, the mice were fasted for 6 hours. Thereafter, the serum was collected. Serum glucose, triglyceride (TG), total cholesterol (TC), aspartate transaminase (AST), and alanine transaminase (ALT) levels were measured using assay kits (ASAN Pharmaceutical, Seoul, Korea). Serum insulin levels were measured using a mouse insulin ELISA kit (Shibayagi, Shibukawa, Japan). The homeostasis model assessment for insulin resistance (HOMA-IR) values were calculated as follows: HOMA-IR $=$ fasting serum glucose $(\mathrm{mmol} / \mathrm{L}) \times$ fasting serum insulin $(\mu \mathrm{U} / \mathrm{mL}) / 22.5$.

\section{Glucose and insulin tolerance tests}

Blood glucose concentrations were measured by placing a small 
drop of blood taken from the tip of the tail on a glucose meter strip (Auto-Check, Diatech Korea Co., Seoul, Korea). Fasting blood glucose was measured after 6 hours of daytime fasting. Glucose tolerance test and insulin tolerance test were performed by intraperitoneally injecting glucose ( $1 \mathrm{~g} / \mathrm{kg}$ body weight) or insulin (0.75 U/kg body weight; Humulin R, Eli Lilly and Company, Indianapolis, IN, USA) solutions, respectively. Blood glucose concentrations were measured at $0,15,30,60,90$, and 120 minutes.

\section{Renal function test}

A 24-hour urine collection was performed for each mouse in a metabolic cage every 4 weeks until the end of the experiment. In the metabolic cages, mice were allowed access to water $a d l i$ bitum, although no access to food was provided. Renal function tests, evaluating urinary albumin, creatinine concentration, and the albumin-creatinine ratio (ACR) were carried out. Creatinine clearance was performed to estimate the glomerular filtration rate (GFR). Creatinine clearance was calculated as urinary creatinine $\times$ urine volume $\times 1,000 /$ serum creatinine $/ 1,440$ and was expressed as $\mu \mathrm{L} / \mathrm{min}$. Urine and plasma creatinine concentrations were assessed using a commercial test kit (Exocell, a division of Ethos Biosciences Inc., Newtown Square, PA, USA).

\section{Histological examination of the glomerulus and vascular morphology}

Paraffin-embedded kidney tissues were stained with hematoxylin and eosin (H\&E) for the examination of the glomeruli and the vascular morphology, and the H\&E-stained sections were visualized, for the determination of glomerular volume, vascular wall thickness, cross-sectional area, and nuclear count of the vascular smooth muscle cells, using a microscope equipped with a charge-coupled device camera at $400 \times$ magnification (Pulnix, Sunnyvale, CA, USA). Glomerular histopathology and renal fibrosis were also investigated by immunohistochemical (IHC) staining for nephrin, fibronectin, type IV collagen, NOX4, and monocyte chemoattractant protein 1 (MCP-1). Additionally, intrarenal RAS component expression was detected by the IHC staining of ACE and angiotensin 1 receptor (AT1R).

\section{Ultrastructural examination of glomerular changes}

The renal cortex was partially excised and immersed in a buffer solution for transmission electron microscopy (TEM). The glomerular basement membrane (GBM) thickness, foot podocyte width, and slit pore density were investigated using TEM at $15,000 \times$ magnification.

\section{Western blot analysis}

Renal expression of nephrin (1:1,000, sc-377246), fibronectin (1:1,000, sc-8422), type IV collagen (1:1,000, sc-11360), ACE (1:1,000, sc-23909), NOX4 (1:1,000, sc-30141), MCP-1 (1:2,000, sc-28879) (all from Santa Cruz Biotech, Santa Cruz, CA, USA), and AT1R (1:1,000, ab-18801) (Abcam, Cambridge, UK) was detected by Western blot analysis. Renal cortex tissues were homogenized in protein lysis buffer. All samples were centrifuged at $13,000 \times \mathrm{g}$ for 20 minutes. The supernatants were mixed with the sample buffer and heated for 5 minutes at $95^{\circ} \mathrm{C}$. Sample aliquots containing 15 to $30 \mu \mathrm{g}$ of protein were loaded on sodium dodecyl sulphate polyacrylamide gel electrophoresis $(8 \%$ to $12 \%$ ) and subsequently transferred to polyvinylidene difluoride membranes. The membranes were incubated at $4{ }^{\circ} \mathrm{C}$ overnight with primary antibodies and washed three times in Tris-buffered saline with $0.1 \%$ Tween 20 . The membranes were then incubated with horseradish peroxidase-conjugated secondary antibodies for 1 hour at room temperature. The gels were visualized using an enhanced chemiluminescence gel electrophoresis system (Amersham Biosciences, Buckinghamshire, UK). The intensity of the bands was measured using ImageJ software version $1.50 \mathrm{i}$ (NIH, Bethesda, MD, USA).

\section{Measurement of systolic blood pressure}

SBP was monitored at the start of the experiment and after every 2 weeks for the duration of the experiment using mouse tailcuff photoplethysmography (BP-2000 blood pressure analysis system, Visitech Systems Inc., Apex, NC, USA). The mice were placed in a restrainer at $34^{\circ} \mathrm{C}$ for 5 minutes. Eight consecutive SBP values were recorded. Furthermore, heart rate was obtained from the data transformation of arterial pulses.

\section{Statistical analysis}

All data are presented as the mean \pm standard error of the mean. Statistical analysis included one-way analysis of variance (ANOVA) and Tukey's post hoc test for multiple comparisons, and was conducted using SPSS Statistics software version 20.0 (IBM Corp., Armonk, NY, USA). Statistical significance was set at $P<0.05$.

\section{RESULTS}

Tetrahydrocurcumin prevents increased body weight and food intake in HFD-fed mice

HFD-fed mice showed a gradual increase in body weight (Fig. 1A) and food intake (Fig. 1B) throughout the experimental peri- 
od. Significant differences in body weight and food intake were observed after 4 and 5 weeks, respectively $(P<0.05$ vs. RD-fed mice). Treatment with THU, particularly at the dose of $100 \mathrm{mg} /$ $\mathrm{kg}$ /day, prevented the increased body weight and food intake $(P<0.05$ vs. HFD+Veh) (Fig. 1$)$.

\section{Tetrahydrocurcumin alleviates glucose intolerance, insulin resistance, and renal dysfunction in HFD-fed mice}

HFD-fed mice exhibited hyperglycemia, insulin resistance, and dyslipidemia (Table 1). THU effectively reduced serum glucose, insulin, TC, TG, AST, and ALT levels in these mice in a dose- dependent manner (Table 1). Moreover, THU potentially attenuated glucose intolerance (Fig. 2A, B) and insulin resistance (Fig. 2C, D) in HFD-fed mice, which is an important observation, as the alteration of metabolic parameters and glucose homeostasis plays a dominant role in the pathogenesis of kidney dysfunction. The present study demonstrated that consumption of HFD increased ACR, kidney weight, and GFR (Fig. 2E-G). Interestingly, treatment with THU, especially at a high dose $(100 \mathrm{mg} /$ $\mathrm{kg} /$ day), significantly decreased these defects $(P<0.05)$. These data suggest that THU has beneficial effects on glucose intolerance, insulin resistance, and kidney function.
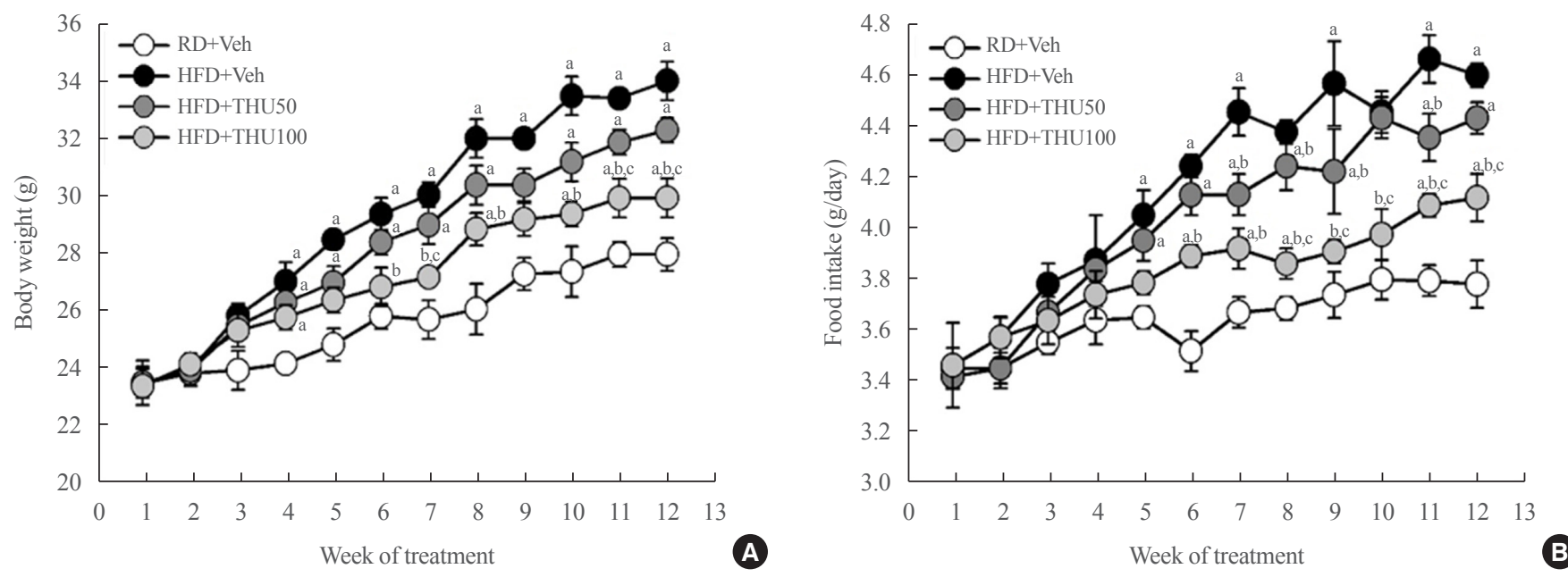

Fig. 1. Tetrahydrocurcumin (THU) prevents an increase in body weight and food intake in high-fat diet (HFD)-fed mice. C57BL/6J mice were fed a regular diet (RD) or HFD for 12 weeks, and THU (50 or $100 \mathrm{mg} / \mathrm{kg} / \mathrm{day})$ was intragastrically administered with HFD. Changes in body weight (A) and food intake (B) during the experimental period. ${ }^{a} P<0.05$ vs. RD+vehicle (Veh); ${ }^{\text {b }} P<0.05$ vs. HFD+Veh $(n=8$ to 10 per group); ${ }^{\mathrm{c}} \mathrm{P}<0.05$ vs. HFD+THU50.

Table 1. Tetrahydrocurcumin Improves Impaired Metabolic Parameters in High-Fat Diet-Fed Mice

\begin{tabular}{lcccc}
\hline & & RD + Veh & \multicolumn{2}{c}{ HFD } \\
\cline { 3 - 4 } Parameter & & Veh & THU50 & THU100 \\
\hline Serum glucose, $\mathrm{mg} / \mathrm{dL}$ & $189.78 \pm 10.99$ & $289.08 \pm 14.23^{\mathrm{a}}$ & $276.47 \pm 15.30^{\mathrm{a}, \mathrm{b}}$ & $254.42 \pm 11.93^{\mathrm{a}, \mathrm{b}, \mathrm{c}}$ \\
Serum insulin, $\mathrm{pg} / \mathrm{mL}$ & $99.80 \pm 8.09$ & $234.31 \pm 15.39^{\mathrm{a}}$ & $202.02 \pm 22.21^{\mathrm{a}, \mathrm{b}}$ & $146.58 \pm 15.14^{\mathrm{a}, \mathrm{b}, \mathrm{c}}$ \\
HOMA-IR index & $1.35 \pm 0.21$ & $5.07 \pm 0.32^{\mathrm{a}}$ & $3.90 \pm 0.08^{\mathrm{a}, \mathrm{b}}$ & $2.56 \pm 0.15^{\mathrm{a}, \mathrm{b}, \mathrm{c}}$ \\
Serum total cholesterol, mg/dL & $167.58 \pm 19.25$ & $323.34 \pm 20.65^{\mathrm{a}}$ & $295.12 \pm 15.39^{\mathrm{a}, \mathrm{b}}$ & $259.10 \pm 11.37^{\mathrm{a}, \mathrm{b}, \mathrm{c}}$ \\
Serum triglycerides, mg/dL & $80.25 \pm 9.25$ & $151.47 \pm 21.39^{\mathrm{a}}$ & $147.64 \pm 18.32^{\mathrm{a}}$ & $119.54 \pm 15.02^{\mathrm{a}, \mathrm{b}, \mathrm{c}}$ \\
Serum AST, unit/mL & $89.34 \pm 10.58$ & $132.76 \pm 12.36^{\mathrm{a}}$ & $128.09 \pm 11.25^{\mathrm{a}}$ & $109.33 \pm 9.20^{\mathrm{a}, \mathrm{b}, \mathrm{c}}$ \\
Serum ALT, unit/mL & $40.39 \pm 3.25$ & $56.08 \pm 4.59^{\mathrm{a}}$ & $45.78 \pm 6.85^{\mathrm{a}, \mathrm{b}}$ & $43.89 \pm 7.51^{\mathrm{a}, \mathrm{b}}$ \\
\hline
\end{tabular}

Values are expressed as mean \pm standard error of the mean.

RD, regular diet; Veh, vehicle; HFD, high-fat diet; THU, tetrahydrocurcumin; HOMA-IR, homeostasis model assessment for insulin resistance; AST, aspartate transaminase; ALT, alanine transaminase.

${ }^{\mathrm{a}} P<0.05$ vs. RD+Veh; ${ }^{\mathrm{b}} P<0.05$ vs. HFD + Veh; ${ }^{\mathrm{c}} P<0.05$ vs. HFD+THU50 (each group, $n=8$ to 10 per group). 

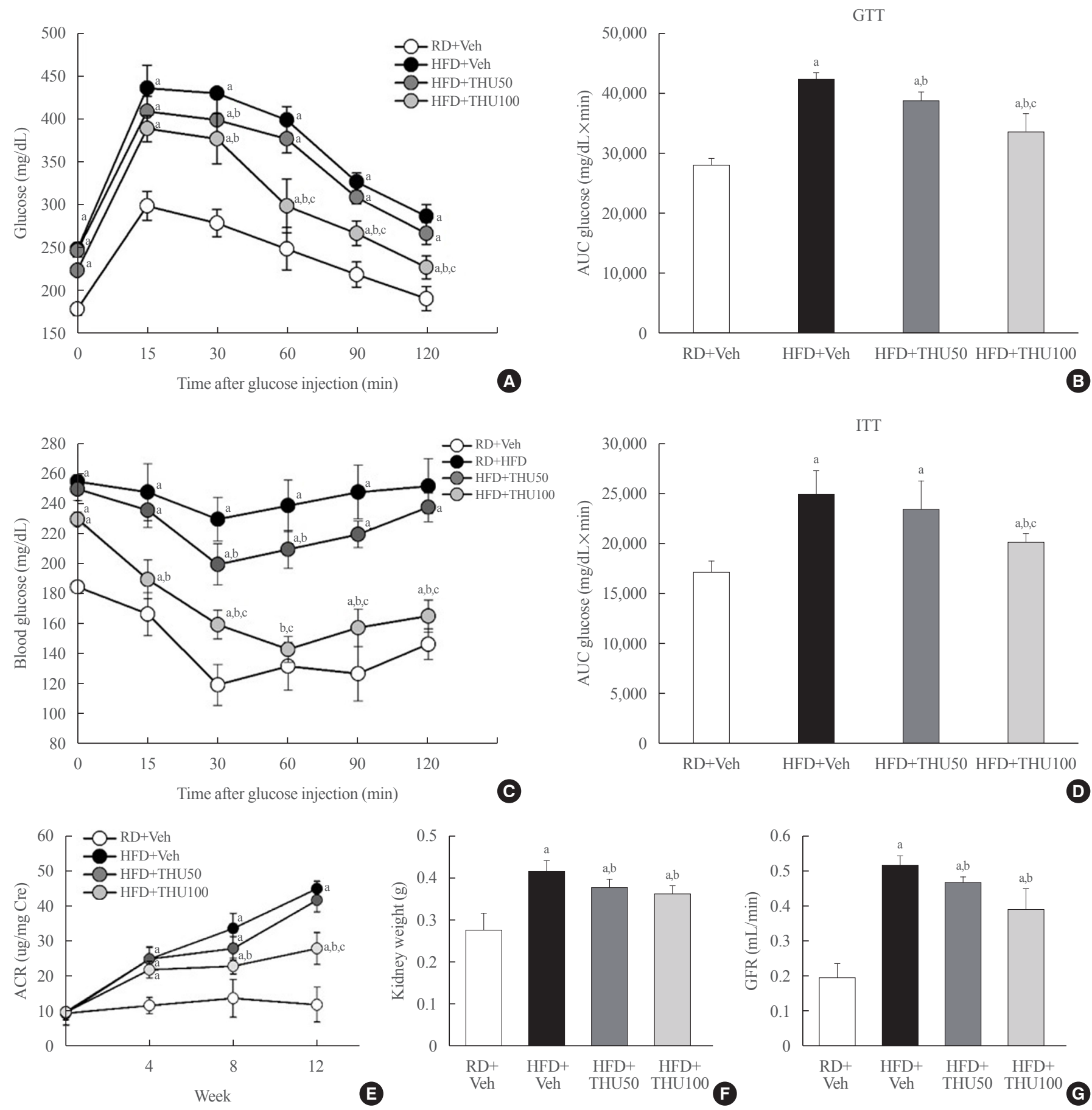

Fig. 2. Tetrahydrocurcumin (THU) alleviates glucose intolerance, insulin resistance, and renal dysfunction in high-fat diet (HFD)-fed mice. Intraperitoneal injection (IP) glucose tolerance test (GTT) and IP insulin tolerance test (ITT) (A, C) at 10 weeks. Levels of area under curve (AUC) in GTT and ITT (B, D). Kidney weight differences among the four groups (E). Albumin-creatinine ratio (ACR) analysis in urine collected at 24 hours (F). Changes in the glomerular filtration rate (GFR) after THU administration (G). RD, regular diet; Veh, vehicle. ${ }^{a} P<0.05$ vs. RD+Veh; ${ }^{\mathrm{b}} P<0.05$ vs. HFD+Veh; ${ }^{\mathrm{c}} P<0.05$ vs. HFD+THU50 ( $n=8$ to 10 per group). 


\section{Tetrahydrocurcumin prevents glomerular hypertrophy in} HFD-fed mice

HFD-induced DN is closely associated with glomerular damage. Histopathological analysis showed glomerular expansion in the HFD-fed mice. However, THU at $100 \mathrm{mg} / \mathrm{kg} /$ day reduced glomerular area and volume (Fig. 3A-D). GBM thickness was followed by mesangial expansion, which is a hallmark of DN. HFD-fed mice exhibited increased GBM thickness, and decreased number of slit pores. However, THU treatment reversed the HFD-induced changes in GBM thickness, slit pore numbers,
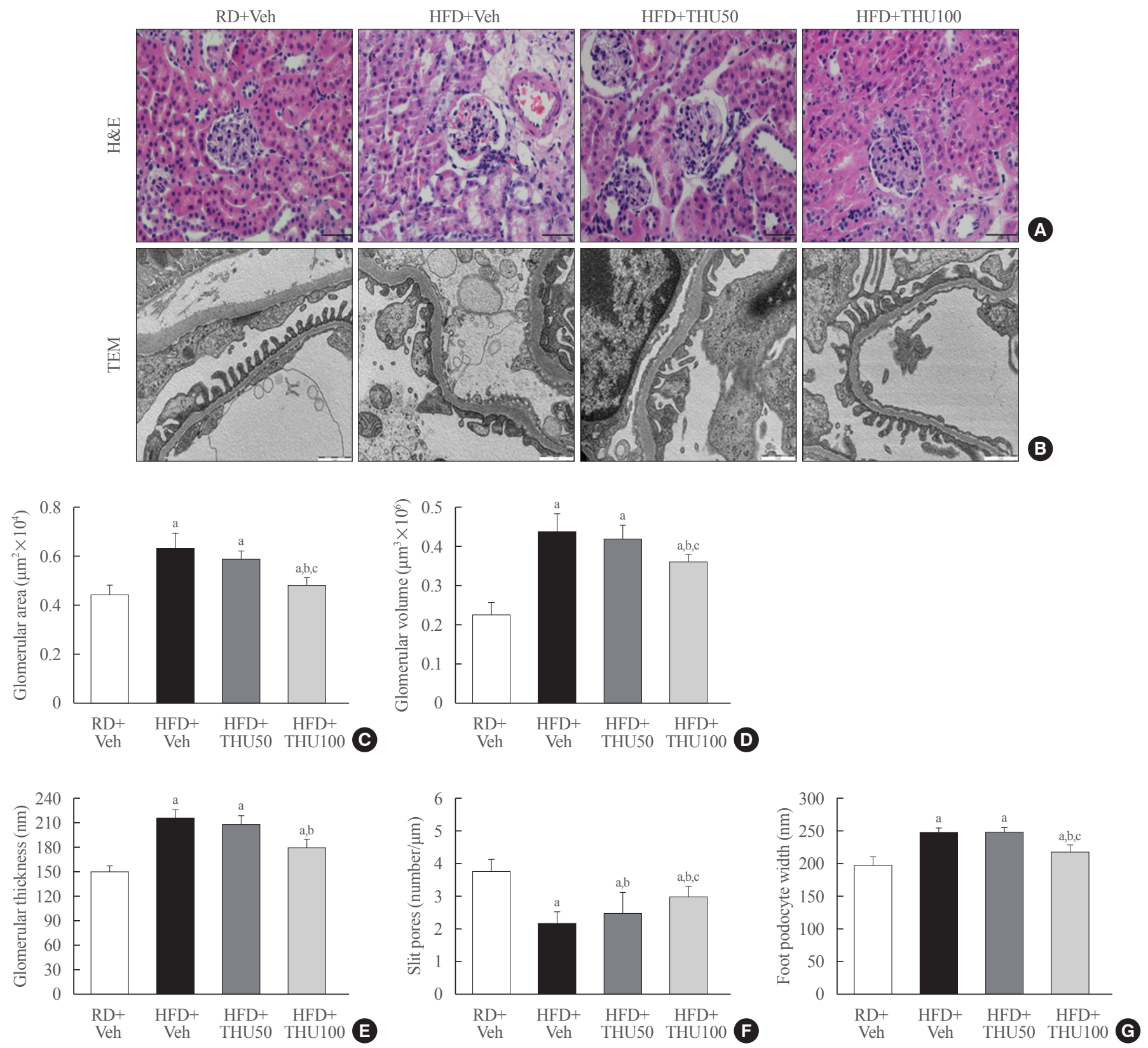

Fig. 3. Tetrahydrocurcumin (THU) protects against glomerular hypertrophy in high-fat diet (HFD)-fed mice. Effect of THU on the enlarged glomeruli of HFD-fed mice. Hematoxylin and eosin staining of the kidneys of all experimental groups (A). Changes in glomerular area and glomerular volume by THU (C, D) ( $n=8$ to 10 per group). Renal ultrastructure changes caused by THU in HFD (B). Quantitative transmission electron microscopy (TEM) data. The glomerular basement membrane (GBM) thickness (E), slit pore numbers (F), and foot process width of podocytes $(\mathrm{G})$ were analyzed by TEM at $15,000 \times$ magnification ( $n=5$ per group). RD, regular diet; Veh, vehicle. ${ }^{\text {a }} P<0.05$ vs. $\mathrm{RD}+\mathrm{Veh} ;{ }^{\mathrm{b}} P<0.05 \mathrm{vs}$. HFD+Veh; ${ }^{\mathrm{c}} P<0.05$ vs. HFD+THU50 (scale bar $=50 \mu \mathrm{m}$ ). 
and the foot width of podocytes, particularly in the THU100 group (Fig. 3E-G). These data suggest that treatment with THU at $100 \mathrm{mg} / \mathrm{kg} /$ day prevented glomerular hypertrophy and the mesangial expansion induced by HFD consumption.

\section{Tetrahydrocurcumin restored nephrin expression and decreased extracellular matrix accumulation in the diabetic glomeruli of HFD-fed mice}

Nephrin is a key molecule that forms the glomerular barrier. Nephrin levels were decreased in the HFD group. Treatment with THU $(100 \mathrm{mg} / \mathrm{kg} /$ day $)$ in HFD-fed mice increased the nephrin levels. It also decreased the expression of fibronectin and collagen IV as indicated by IHC staining (Fig. 4A-D), and Western blot assays (Fig. 4E, F). This suggests that THU at a dose of $100 \mathrm{mg} / \mathrm{kg} /$ day prevents the accumulation of extracellular matrix (ECM).

\section{Tetrahydrocurcumin downregulates ACE-AT1R-NOX4- MCP-1 expression in the glomeruli of HFD-fed mice}

The mechanisms of hypertension, oxidative stress, and renal inflammation in HFD-fed mice include the activation of intrarenal RAS, nicotinamide adenine dinucleotide phosphate (NADPH) oxidase activity, and MCP-1. In the glomeruli of the HFD-fed mice, an increase in the stained areas denoting ACE and AT1R expression was observed. Further, expression of NADPH oxidase 4 and of inflammatory signals, such as MCP-1, was also increased, as indicated by IHC staining (Fig. 5A-D). However, the administration of THU100 resulted in a decrease in the positively stained areas corresponding with the aforementioned markers (Fig. 5E). The protein expression levels of ACE, AT1R, NOX4, and MCP-1 in the kidney were consistent with the IHC staining data (Fig. 5F). These results suggest that THU at a dose of $100 \mathrm{mg} / \mathrm{kg}$ /day reduces the activity of RAS, and reactive oxygen species (ROS), thus attenuating renal inflammation.

\section{Tetrahydrocurcumin attenuates high systolic blood pressure and the remodeling of vascular structures in HFD-fed mice}

SBP was elevated, and vascular structures were altered by the long-term administration of HFD. HFD increased the number of nuclei on the tunica surface, the wall thickness, and the crosssectional area of the blood vessels (Fig. 6A-D). Administration of THU (100 mg/kg/day) reduced the SBP (Fig. 6E). However, the heart rate did not change in any of the groups (Fig. 6F). These data suggest that THU100 prevents vascular remodeling, thereby preventing an increase in SBP.

\section{DISCUSSION}

The present study showed that treatment with THU at a dose of $100 \mathrm{mg} / \mathrm{kg} /$ day effectively prevented kidney injury and high SBP in HFD-induced type 2 diabetic mice. In this present study, the administration of an HFD for 12 weeks caused type 2 diabetes in mice, as evidenced by the observation of weight gain, impaired glucose regulation, and insulin resistance, and of associated metabolic disorders, including hyperinsulinemia and dyslipidemia. Albuminuria, glomerular hypertrophy, thickening of the GBM, and podocyte foot detachment were also noted in HFD-fed mice, suggesting that the kidneys were damaged and enriched in ECM components such as collagen and fibronectin. Importantly, HFD consumption increased SBP and induced vascular remodeling. These findings are consistent with a previous study reporting that long-term feeding of HFD for 12 weeks induced obesity, glomerular damage, and vascular structural changes [25].

The occurrence of albuminuria, hypertension, and histopathological changes in the glomeruli of diabetes patients are characteristics of $\mathrm{DN}$, which is a progressive kidney disease. A damaged kidney loses its physiological functions, including that of filtration and reabsorption [26,27], resulting in leakage of albumin into the urine and the retention of waste products in the body.

HFD consumption may exert a deleterious effect on the kidneys by promoting renal inflammation. HFD increases the infiltration of macrophages in adipose tissue and kidneys, leading to an inflammatory response and tissue damage. In addition, HFD may induce kidney injury by promoting glomerular hypertrophy, the thickening of the GBM, and glomerular sclerosis, thereby disrupting renal hemodynamics. Our results are consistent with those of a previous study showing that renal vasodilation, increased renal blood flow, and glomerular hyperfiltration occurred after weight gain [28].

Hyperglycemia is a consequence of HFD-induced type 2 diabetes. It plays an important role in the pathogenesis of kidney injury. Oxidative stress is a well-known pathogenic mechanism of hyperglycemia, which triggers diabetic complications. Oxidative stress triggers the accumulation of angiotensinogen, subsequently activating the RAS [29]. A previous study revealed that all RAS components (ACE, angiotensin II, and AT1R) are extensively expressed in the renal endothelial cells of diabetic rodents [30]. Moreover, ACE activity levels increase along with blood glucose and hemoglobin Alc levels in diabetes patients with nephropathy [31]. These studies suggest that hyperglyce- 

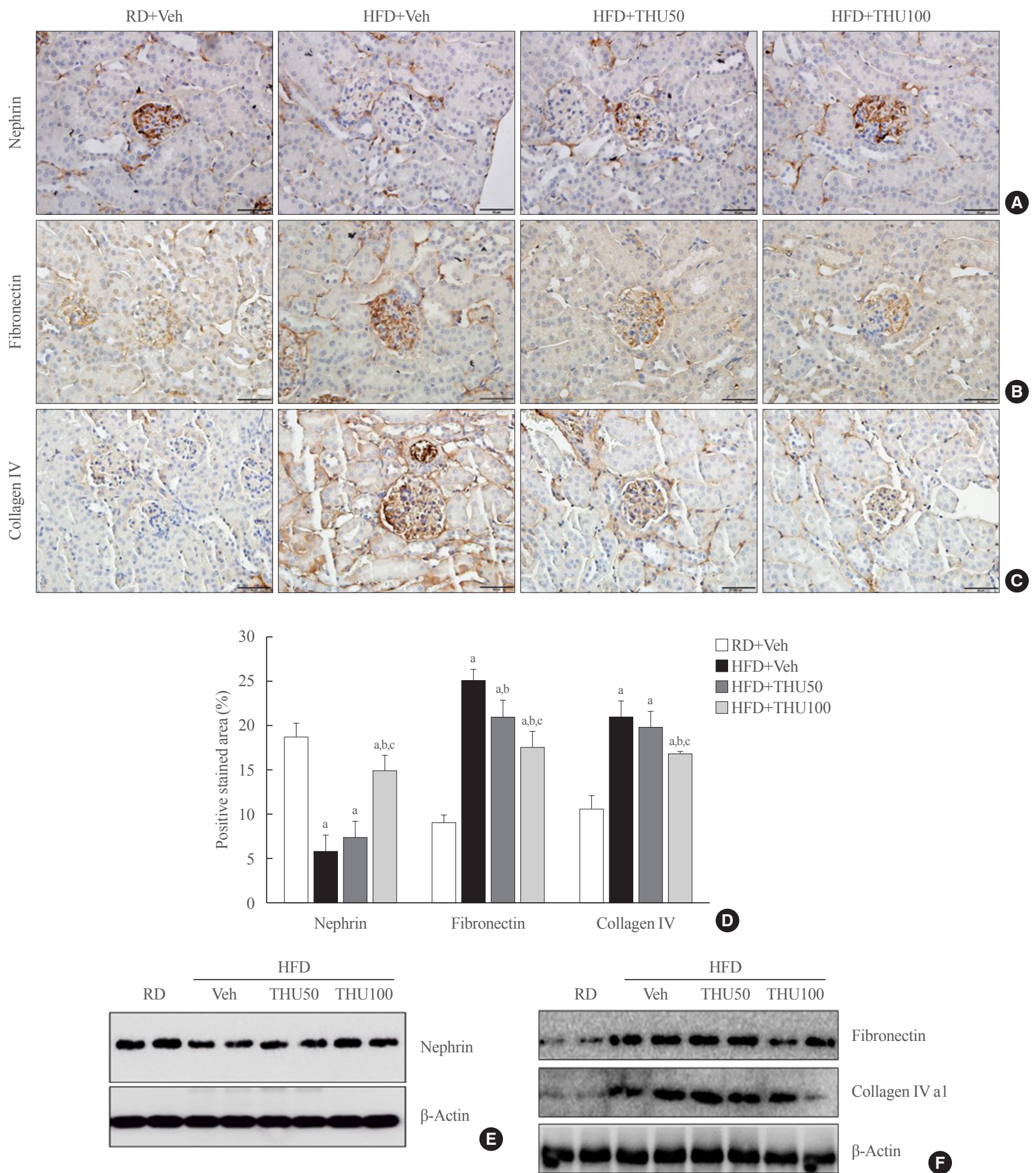

Fig. 4. Tetrahydrocurcumin (THU) restores nephrin expression and decreases extracellular matrix accumulation in the diabetic glomeruli of high-fat diet (HFD)-fed mice. Renal nephrin (A), fibronectin (B), and collagen type IV (C) were subjected to immunohistochemical (IHC) staining. Panel (D) indicates the percentage of positively stained area of each protein in glomeruli. Original magnification was $400 \times$ (scale bar $=50 \mu \mathrm{m}$ ). Nephrin, fibronectin, and type IV collagen expression were analyzed by Western blotting (E, F). All protein expression analyses using Western blotting were performed on samples of the renal cortex ( $n=8$ to 10 per group). RD, regular diet; Veh, vehicle. ${ }^{a} P<0.05$ vs. $\mathrm{RD}+\mathrm{Veh} ;{ }^{\mathrm{b}} P<0.05$ vs. HFD+Veh; ${ }^{\mathrm{c}} P<0.05$ vs. HFD+THU50. 

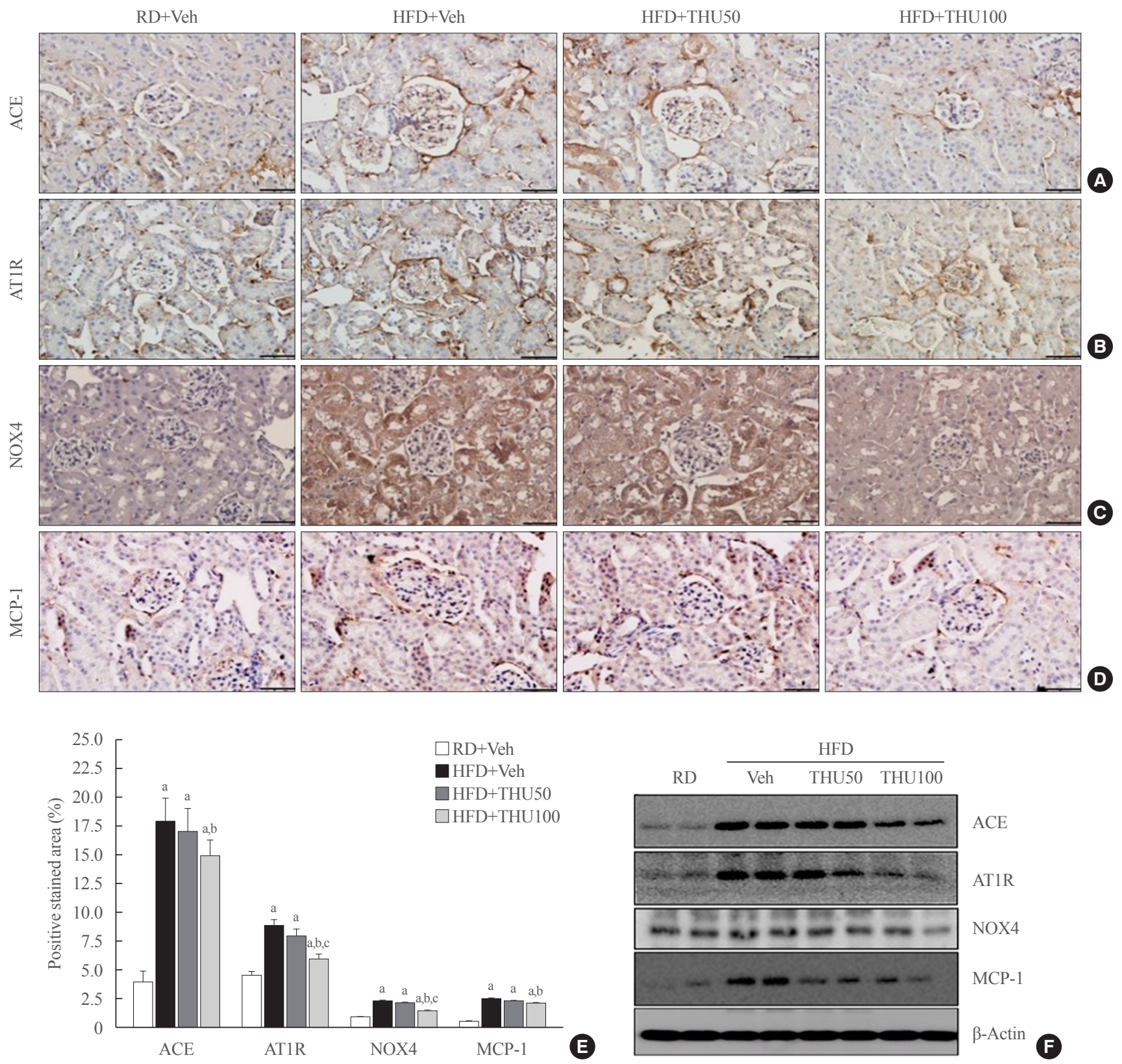

Fig. 5. Tetrahydrocurcumin (THU) downregulates the expression of angiotensin-converting enzyme (ACE), angiotensin 1 receptor (AT1R), nicotinamide adenine dinucleotide phosphate (NADPH) oxidase 4 (NOX4), and chemokine C-C motif ligand 2 (MCP-1) in the glomeruli of high-fat diet (HFD)-fed mice. (A, B, C, D) ACE, AT1R, NADPH oxidase 4, and MCP-1 expression indicated by immunohistochemical staining. (E) Panel indicates the positively stained area of each protein in the glomeruli ( $n=4$ to 5 per group; scale bar $=50 \mu \mathrm{m})$. (F) Western blot analysis of the renal cortex. RD, regular diet; Veh, vehicle. ${ }^{\mathrm{a}} P<0.05 \mathrm{vs}$. RD+Veh; ${ }^{\mathrm{b}} P<0.05 \mathrm{vs}$. HFD+Veh; ${ }^{\mathrm{c}} P<0.05$ vs. HFD+THU50.

mia activates the RAS. The binding of angiotensin II to AT1R activates NADPH oxidase (NOX), which transfers an electron from NADPH to $\mathrm{O}_{2}$, producing the superoxide anion $\left(\mathrm{O}_{2}{ }^{-}\right)$[32]. Excess $\mathrm{O}_{2}{ }^{-}$generation causes oxidative stress and oxidative damage to renal tissues. Moreover, NOX-derived ROS were found to be involved in angiotensin II-induced MCP-1 expres- sion in mesangial cells [33].

We demonstrated that THU alleviates kidney injury by suppressing the RAS/NOX4/MCP-1 axis. The possible mechanism of action of THU might involve its antioxidant property and blood glucose-lowering effect. The structure of THU includes a phenol and a $\beta$-diketone functional group, which is a common 


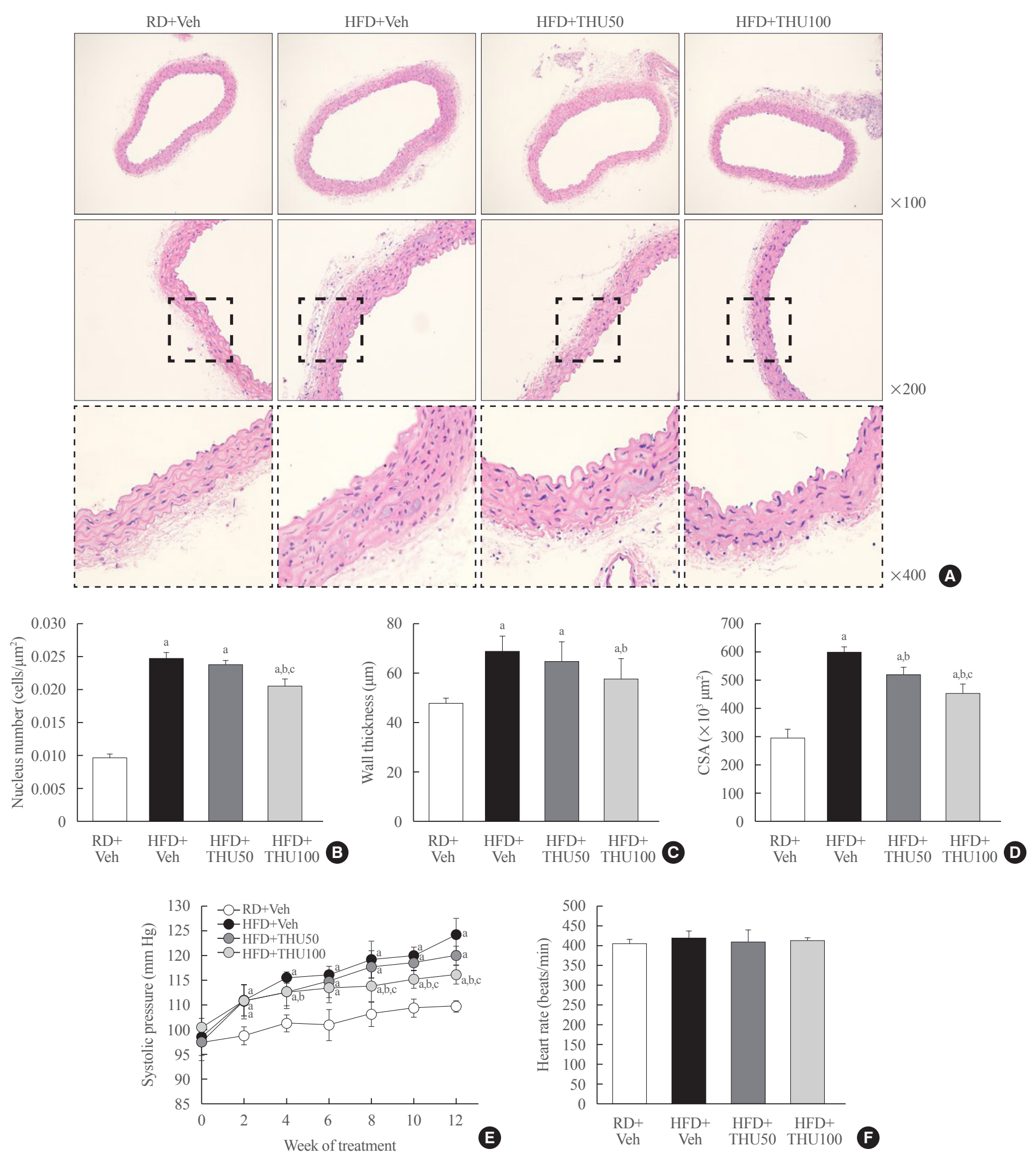

Fig. 6. Tetrahydrocurcumin (THU) attenuates high systolic blood pressure and remodeling of vascular structures in high-fat diet (HFD)-fed mice. Vascular structural changes (A), nucleus count by tunica media area $\left(\mu \mathrm{m}^{2}\right)$, vascular wall thickening $(\mu \mathrm{m})$, vascular cross-sectional area $(\mathrm{CSA})\left(\times 10^{3} \mu \mathrm{m}^{2}\right)(\mathrm{B}, \mathrm{C}, \mathrm{D})$, systolic blood pressure $(\mathrm{E})$, and heart rate throughout the 12 weeks of treatment $(\mathrm{F})(n=8$ to 10 per group). RD, regular diet; Veh, vehicle. ${ }^{\mathrm{a}} P<0.05$ vs. RD+Veh; ${ }^{\mathrm{b}} P<0.05$ vs. HFD+Veh; ${ }^{\mathrm{c}} P<0.05$ vs. HFD+THU50. 
structural feature found in antioxidative molecules [34]. Previous studies have demonstrated that the administration of THU mitigates oxidative stress by scavenging $\mathrm{O}_{2}^{-}$[35]. Thus, the subsequent reduction in the excessive levels of ROS (under the hyperglycemic conditions) potentially suppresses the activation of the RAS/NOX4/MCP-1 axis.

Apart from hyperglycemia-mediated ROS that enhances RAS activation, hyperglycemia directly activates the RAS [30]. The present study showed the beneficial effects of THU for improving insulin sensitivity, and thereby lowering serum glucose levels. The effect of THU on the reduction of hyperglycemia may be partially explained by the antioxidant properties of THU. Attenuation of oxidative stress is directly associated with increased insulin sensitivity by reducing the oxidation of insulin receptors and intracellular glucose transporters [36]. This suggests that THU may prevent the alteration of insulin receptor signaling. Our findings are consistent with those of previous studies showing improved insulin sensitivity in diabetic mice after THU treatment $[37,38]$. Therefore, attenuation of hyperglycemia alters the activation of the RAS/NOX4/MCP-1 axis under diabetic conditions.

Administration of HFD for 12 weeks induced glomerular hypertrophy and podocyte injury. Treatment with THU at $100 \mathrm{mg} /$ $\mathrm{kg}$ /day in HFD-fed mice reduced the foot process width of podocytes, determined morphometrically by electron microscopy, suggesting that THU potentially alleviates podocyte foot process effacement. Podocyte dysfunction is associated with altered insulin signaling pathways [39]. Moreover, treatment with ACE inhibitors or angiotensin II type 1-receptor blockers in patients with DN can reduce podocyte injury [40]. This confirms that the activation of the RAS is implicated in podocyte injury. Furthermore, oxidative stress plays a major role in podocyte injury by enhancing endoplasmic reticulum stress, which may trigger autophagy [41]. The mechanism by which THU alleviates alterations in podocyte function may be associated with the antioxidant properties of THU.

Insulin sensitivity is important for normal podocyte function and glucose regulation. In this study, HFD-fed mice showed insulin resistance and increased HOMA-IR and hyperglycemia; however, this effect was attenuated by treatment with THU does dependently. Oxidative stress-associated obesity and increased inflammation potentially alter insulin action by worsening the insulin receptor interaction and signal transduction. This phenomenon is a leading cause of insulin resistance and hyperinsulinemia. Attenuation of oxidative stress by THU prevents the alteration of insulin receptor signaling, thereby improving insulin sensitivity [36].

THU attenuates high SBP in HFD-fed mice by reducing aortic wall thickness. This effect may be related to the antioxidant properties of THU. The reduction in oxidative stress increases the bioavailability of nitric oxide, a potent vasodilator, leading to increased vascular relaxation and a reduction in SBP.

The cumulative data showed that THU can serve an effective bioactive compound for the attenuation of DN due to its ability to reduce metabolic abnormalities, kidney injury, and the high SBP caused by HFD consumption.

In conclusion, THU exerts a renoprotective effect in HFD-fed mice by inhibiting the intrarenal RAS /NOX4/MCP-1 axis and attenuating insulin resistance and reducing the high SBP.

\section{CONFLICTS OF INTEREST}

No potential conflict of interest relevant to this article was reported.

\section{ACKNOWLEDGMENTS}

This work was supported by the National Research Foundation of Korea (NRF) grant funded by the Korean government (MSIT) (2018R1A2B6005360, 2020R1A2C2003438, 2021R1A2B5B01002354). Tetrahydrocurcumin was generously provided by the Government Pharmaceutical Organization, Bangkok, Thailand.

\section{AUTHOR CONTRIBUTIONS}

Conception or design: W.S., E.S.L., H.M.K. Acquisition, analysis, or interpretation of data: W.S., K.B.H. Drafting the work or revising: W.S., K.B.H. Final approval of the manuscript: U.K., E.Y.L., C.H.C.

\section{ORCID}

Weerapon Sangartit https://orcid.org/0000-0002-5058-2294

Kyung Bong Ha https://orcid.org/0000-0002-6903-7253

Choon Hee Chung https://orcid.org/0000-0003-1144-7206

\section{REFERENCES}

1. Vasavada N, Agarwal R. Role of oxidative stress in diabetic nephropathy. Adv Chronic Kidney Dis 2005;12:146-54.

2. Rosenbaum M, Leibel RL. The physiology of body weight 
regulation: relevance to the etiology of obesity in children. Pediatrics 1998;101(3 Pt 2):525-39.

3. Hall JE, Crook ED, Jones DW, Wofford MR, Dubbert PM. Mechanisms of obesity-associated cardiovascular and renal disease. Am J Med Sci 2002;324:127-37.

4. Frohlich ED. Clinical management of the obese hypertensive patient. Cardiol Rev 2002;10:127-38.

5. Ledwozyw A, Michalak J, Stepien A, Kadziolka A. The relationship between plasma triglycerides, cholesterol, total lipids and lipid peroxidation products during human atherosclerosis. Clin Chim Acta 1986;155:275-83.

6. Kotsis VT, Stabouli SV, Papamichael CM, Zakopoulos NA. Impact of obesity in intima media thickness of carotid arteries. Obesity (Silver Spring) 2006;14:1708-15.

7. Wong LK. Global burden of intracranial atherosclerosis. Int J Stroke 2006;1:158-9.

8. Kincaid-Smith P. Hypothesis: obesity and the insulin resistance syndrome play a major role in end-stage renal failure attributed to hypertension and labelled 'hypertensive nephrosclerosis'. J Hypertens 2004;22:1051-5.

9. Serra A, Romero R, Lopez D, Navarro M, Esteve A, Perez N, et al. Renal injury in the extremely obese patients with normal renal function. Kidney Int 2008;73:947-55.

10. Gallagher S. The challenges of obesity and skin integrity. Nurs Clin North Am 2005;40:325-35.

11. Tonneijck L, Muskiet MH, Smits MM, van Bommel EJ, Heerspink HJ, van Raalte DH, et al. Glomerular hyperfiltration in diabetes: mechanisms, clinical significance, and treatment. J Am Soc Nephrol 2017;28:1023-39.

12. Thallas-Bonke V, Jandeleit-Dahm KA, Cooper ME. Nox-4 and progressive kidney disease. Curr Opin Nephrol Hypertens 2015;24:74-80.

13. Hall JE, do Carmo JM, da Silva AA, Wang Z, Hall ME. Obesity, kidney dysfunction and hypertension: mechanistic links. Nat Rev Nephrol 2019;15:367-85.

14. Peterson JC, Adler S, Burkart JM, Greene T, Hebert LA, Hunsicker LG, et al. Blood pressure control, proteinuria, and the progression of renal disease. The modification of diet in renal disease study. Ann Intern Med 1995;123:754-62.

15. Viazzi F, Leoncini G, Pontremoli R. Antihypertensive treatment and renal protection: the role of drugs inhibiting the renin-angiotensin-aldosterone system. High Blood Press Cardiovasc Prev 2013;20:273-82.

16. Mogensen CE, Neldam S, Tikkanen I, Oren S, Viskoper R, Watts RW, et al. Randomised controlled trial of dual blockade of renin-angiotensin system in patients with hyperten- sion, microalbuminuria, and non-insulin dependent diabetes: the candesartan and lisinopril microalbuminuria (CALM) study. BMJ 2000;321:1440-4.

17. Go YM, Jones DP. Redox compartmentalization in eukaryotic cells. Biochim Biophys Acta 2008;1780:1273-90.

18. Cooper ME, Mundel P, Boner G. Role of nephrin in renal disease including diabetic nephropathy. Semin Nephrol 2002;22:393-8.

19. Sarafidis PA, Khosla N, Bakris GL. Antihypertensive therapy in the presence of proteinuria. Am J Kidney Dis 2007;49: 12-26.

20. Murugan P, Pari L. Antioxidant effect of tetrahydrocurcumin in streptozotocin-nicotinamide induced diabetic rats. Life Sci 2006;79:1720-8.

21. Nakmareong S, Kukongviriyapan U, Pakdeechote P, Donpunha W, Kukongviriyapan V, Kongyingyoes B, et al. Antioxidant and vascular protective effects of curcumin and tetrahydrocurcumin in rats with L-NAME-induced hypertension. Naunyn Schmiedebergs Arch Pharmacol 2011;383:51929.

22. Zhang XX, Kong J, Yun K. Prevalence of diabetic nephropathy among patients with type 2 diabetes mellitus in China: a meta-analysis of observational studies. J Diabetes Res 2020;2020:2315607.

23. Zhang ZB, Luo DD, Xie JH, Xian YF, Lai ZQ, Liu YH, et al. Curcumin's metabolites, tetrahydrocurcumin and octahydrocurcumin, possess superior anti-inflammatory effects in vivo through suppression of TAK1-NF- $\kappa$ B pathway. Front Pharmacol 2018;9:1181.

24. Noshahr ZS, Salmani H, Khajavi Rad A, Sahebkar A. Animal models of diabetes-associated renal injury. J Diabetes Res 2020;2020:9416419.

25. Masters SL, Latz E, O'Neill LA. The inflammasome in atherosclerosis and type 2 diabetes. Sci Transl Med 2011;3: $81 \mathrm{ps} 17$.

26. Boini KM, Xia M, Abais JM, Li G, Pitzer AL, Gehr TW, et al. Activation of inflammasomes in podocyte injury of mice on the high fat diet: effects of ASC gene deletion and silencing. Biochim Biophys Acta 2014;1843:836-45.

27. Hunley TE, Ma LJ, Kon V. Scope and mechanisms of obesity-related renal disease. Curr Opin Nephrol Hypertens 2010; 19:227-34.

28. Kalantar-Zadeh K, Kopple JD. Obesity paradox in patients on maintenance dialysis. Contrib Nephrol 2006;151:57-69.

29. Kamiyama M, Urushihara M, Morikawa T, Konishi Y, Imanishi M, Nishiyama A, et al. Oxidative stress/angioten- 
sinogen/renin-angiotensin system axis in patients with diabetic nephropathy. Int J Mol Sci 2013;14:23045-62.

30. Chen CM, Juan SH, Chou HC. Hyperglycemia activates the renin-angiotensin system and induces epithelial-mesenchymal transition in streptozotocin-induced diabetic kidneys. J Renin Angiotensin Aldosterone Syst 2018;19:1470320318803009.

31. Ustundag B, Canatan H, Cinkilinc N, Halifeoglu I, Bahcecioglu IH. Angiotensin converting enzyme (ACE) activity levels in insulin-independent diabetes mellitus and effect of ACE levels on diabetic patients with nephropathy. Cell Biochem Funct 2000;18:23-8.

32. Micakovic T, Papagiannarou S, Clark E, Kuzay Y, Abramovic K, Peters J, et al. The angiotensin II type 2 receptors protect renal tubule mitochondria in early stages of diabetes mellitus. Kidney Int 2018;94:937-50.

33. Lee YW, Lee WH, Kim PH. Role of NADPH oxidase in interleukin-4-induced monocyte chemoattractant protein-1 expression in vascular endothelium. Inflamm Res 2010;59: 755-65.

34. Wu JC, Lai CS, Badmaev V, Nagabhushanam K, Ho CT, Pan MH. Tetrahydrocurcumin, a major metabolite of curcumin, induced autophagic cell death through coordinative modulation of PI3K/Akt-mTOR and MAPK signaling pathways in human leukemia HL-60 cells. Mol Nutr Food Res 2011;55:1646-54.

35. Sangartit W, Kukongviriyapan U, Donpunha W, Pak- deechote P, Kukongviriyapan V, Surawattanawan P, et al. Tetrahydrocurcumin protects against cadmium-induced hypertension, raised arterial stiffness and vascular remodeling in mice. PLoS One 2014;9:e114908.

36. Yaribeygi H, Sathyapalan T, Atkin SL, Sahebkar A. Molecular mechanisms linking oxidative stress and diabetes mellitus. Oxid Med Cell Longev 2020;2020:8609213.

37. Chen JW, Kong ZL, Tsai ML, Lo CY, Ho CT, Lai CS. Tetrahydrocurcumin ameliorates free fatty acid-induced hepatic steatosis and improves insulin resistance in HepG2 cells. J Food Drug Anal 2018;26:1075-85.

38. Pari L, Murugan P. Effect of tetrahydrocurcumin on blood glucose, plasma insulin and hepatic key enzymes in streptozotocin induced diabetic rats. J Basic Clin Physiol Pharma$\operatorname{col} 2005 ; 16: 257-74$.

39. Jauregui A, Mintz DH, Mundel P, Fornoni A. Role of altered insulin signaling pathways in the pathogenesis of podocyte malfunction and microalbuminuria. Curr Opin Nephrol Hypertens 2009;18:539-45.

40. Reiser J, Mundel P. Dual effects of RAS blockade on blood pressure and podocyte function. Curr Hypertens Rep 2007; 9:403-8.

41. Yuan Y, Xu X, Zhao C, Zhao M, Wang H, Zhang B, et al. The roles of oxidative stress, endoplasmic reticulum stress, and autophagy in aldosterone/mineralocorticoid receptor-induced podocyte injury. Lab Invest 2015;95:1374-86. 